\title{
Article \\ Nanocone-Shaped Carbon Nanotubes Field-Emitter Array Fabricated by Laser Ablation
}

\author{
Jiuzhou Zhao ${ }^{1,2}$, Zhenjun $\mathrm{Li}^{2,3}$, Matthew Thomas Cole ${ }^{4} \mathbb{D}_{\text {, Aiwei Wang }}{ }^{2,5}$, Xiangdong Guo ${ }^{2,5}$, Xinchuan Liu ${ }^{2}$, \\ Wei Lyu ${ }^{1,2}$, Hanchao Teng 2,5, Yunpeng Qv ${ }^{2,5}$, Guanjiang Liu ${ }^{2}$, Ke Chen ${ }^{2,5}$, Shenghan Zhou ${ }^{2,5}$, Jianfeng Xiao ${ }^{2}$, \\ Yi Li ${ }^{1,6, *}$, Chi $\mathrm{Li}^{2,5, *}$ and Qing Dai ${ }^{2,5, *}$
}

1 Tianjin Key Laboratory of Molecular Optoelectronic Sciences, Department of Chemistry, School of Science, Tianjin University \& Collaborative Innovation Center of Chemical Science and Engineering (Tianjin), Tianjin 300072, China; zhaojz2020@nanoctr.cn (J.Z.); lvw0817@163.com (W.L.)

2 CAS Key Laboratory of Nanophotonic Materials and Devices, CAS Key Laboratory of Standardization and Measurement for Nanotechnology, CAS Center for Excellence in Nanoscience, National Center for Nanoscience and Technology, Beijing 100190, China; lizhenjun@nanoctr.cn (Z.L.); wangaw2020@nanoctr.cn (A.W.); guoxd@nanoctr.cn (X.G.); liuxc2019@nanoctr.cn (X.L.); tenghc2019@nanoctr.cn (H.T.); quyp2020@nanoctr.cn (Y.Q.); liugj20101120@163.com (G.L.); chenke@nanoctr.cn (K.C.); zhoushenghan@nanoctr.cn (S.Z.); xiaojf2020@nanoctr.cn (J.X.)

3 GBA Research Innovation Institute for Nanotechnology, Guangzhou 510700, China

4 Department of Electronic and Electrical Engineering, University of Bath, Bath BA2 7AY, UK; mtc47@bath.ac.uk

5 Center of Materials Science and Optoelectronics Engineering, University of Chinese Academy of Sciences, Beijing 100049, China

6 Joint School of National University of Singapore and Tianjin University, Fuzhou International Campus, Tianjin University, Binhai New City, Fuzhou 350207, China

check for
updates

Citation: Zhao, J.; Li, Z.; Cole, M.T.; Wang, A.; Guo, X.; Liu, X.; Lyu, W.;

Teng, H.; Qv, Y.; Liu, G.; et al.

Nanocone-Shaped Carbon Nanotubes Field-Emitter Array Fabricated by Laser Ablation. Nanomaterials 2021, 11, 3244. https://doi.org/10.3390/ nano11123244

Academic Editor: Filippo Giubileo

Received: 7 November 2021

Accepted: 25 November 2021

Published: 29 November 2021

Publisher's Note: MDPI stays neutral with regard to jurisdictional claims in published maps and institutional affiliations.

Copyright: (c) 2021 by the authors. Licensee MDPI, Basel, Switzerland. This article is an open access article distributed under the terms and conditions of the Creative Commons Attribution (CC BY) license (https:/ / creativecommons.org/licenses/by/ $4.0 /)$.
* Correspondence: liyi@tju.edu.cn (Y.L.); lichi@nanoctr.cn (C.L.); daiq@nanoctr.cn (Q.D.)

\begin{abstract}
The nanocone-shaped carbon nanotubes field-emitter array (NCNA) is a near-ideal fieldemitter array that combines the advantages of geometry and material. In contrast to previous methods of field-emitter array, laser ablation is a low-cost and clean method that does not require any photolithography or wet chemistry. However, nanocone shapes are hard to achieve through laser ablation due to the micrometer-scale focusing spot. Here, we develop an ultraviolet (UV) laser beam patterning technique that is capable of reliably realizing NCNA with a cone-tip radius of $\approx 300 \mathrm{~nm}$, utilizing optimized beam focusing and unique carbon nanotube-light interaction properties. The patterned array provided smaller turn-on fields (reduced from 2.6 to $1.6 \mathrm{~V} / \mu \mathrm{m}$ ) in emitters and supported a higher (increased from 10 to $140 \mathrm{~mA} / \mathrm{cm}^{2}$ ) and more stable emission than their unpatterned counterparts. The present technique may be widely applied in the fabrication of high-performance CNTs field-emitter arrays.
\end{abstract}

Keywords: nanocone array; field emission; carbon nanotubes; laser ablation

\section{Introduction}

Vertically aligned carbon nanotubes (VACNT) provide a much simpler, cheaper, as well as more scalable, manufacturable, and deployable platform upon which to build future electron sources. A variety of methods have come to the fore to achieve VACNT fieldemitter arrays with well-defined patterns, which include the pre-patterning, commonly by nanostamping [1], electron beam lithography [2], or photolithography [3] of the catalyst layer. Although very successful, all these approaches are costly and require time-consuming additional process steps [4] before the CNT growth. Moreover, it is difficult to achieve large length-to-diameter ratios of $>8$, and it is not possible to realize more diverse shapes that are patterned in the out-of-plane direction, such as structures with nanoscale tips. Fundamentally new approaches are required to fabricate VACNT array with nanoscale tips (nanocone) that can be achieved at low cost and high throughput. 
Post-growth, laser ablation patterning is one exciting solution that satisfies the above requirements. Laser processing is a technology that uses the interaction between laser and material to cut, weld, surface treatment, micromachining, and so on [5-12]. The photons of low wavelength ultraviolet (UV) light have high energy, and the single photons of high energy $(>3 \mathrm{eV})$ can directly break (requires 3-10 eV) the chemical bonds of materials (photolytic process) [10]. This is a highly nonlinear process, which make it possible to process materials with sharp edges. In addition, the almost perfect absorption of VACNTs $[13,14]$ for light in a wide wavelength range further reduces the heat-affected zone in the processing process [15]. These factors together make it possible for the laser to process in pattern micron or even nanoscale CNTs structures, which have high and stable current under low electric field intensity.

Here, to create functionally enhanced CNT arrays, we report an ultraviolet laser patterning technique that is capable of realizing a range of well-defined emitter morphologies by accurately adjusting the energy output state and action time of laser to VACNT. At the optimized condition, nanocone-shaped carbon nanotube arrays (NCNAs) were achieved. Laser-processed samples showed up to a $1 \mathrm{~V} / \mu \mathrm{m}$ decrease in the turn-on electric field, more than a $97 \times$ increase in current density over the unpatterned counterparts, and improved stability (the current decay rate was reduced by more than $20 \%$ ). To explore the impacts of the microscale geometry on the emission characteristics of the VACNT thin films, three-dimensional finite element simulations were also undertaken on unpatterned and patterned emitters.

\section{Experimental}

In brief, macroscale $2 \mathrm{~mm} \times 2 \mathrm{~mm}$ square monoliths of VACNT thin films, which were ready for subsequent UV laser processing were synthesized on silicon substrate by PECVD (plasma-enhanced chemical vapor deposition) [11,16-18]. Respectively, as shown in Figure 1a,b, femtosecond laser processing has been demonstrated elsewhere though commonly at different wavelengths such as $800 \mathrm{~nm}$ and $1064 \mathrm{~nm}$ [19-21]; however, in the present work, a nanosecond ultraviolet laser processing platform $(\lambda=355 \mathrm{~nm}$, Suzhou Delong laser Co. Ltd., Suzhou, China; FP-D-DZS-001) was used. AutoCAD (Version 2018, Autodesk, Inc., Mill Valley, CA, USA) software was used in this work to create DGW files required by the laser patterning system. The CAD drawings outline the path of the laser in two dimensions. The coordination of various parameters determines the width, depth, shape, continuity, and smoothness of the ablation groove, which together with the CAD drawings determines the shape of the VACNT created by the laser. (Supplementary Materials Figure S1 shows the arrays with high aspect ratio. Supplementary Materials Figure S2 shows CAD drawings and the corresponding processed shapes).

It has been shown elsewhere that micro and macro-scale geometries within CNT arrays enhanced their field electron emission performances [19-21]. Thus, here, we explored the use of the developed patterning technique to engineer new electron emission sources. Cubic arrays with sides of $20 \mu \mathrm{m}$ and spacing of $40 \mu \mathrm{m}$ were first fabricated on the VACNT film; then, we used this as a benchmark to continuously reduce the size of the tip and fabricated the cone arrays with the tip size of about $15 \mu \mathrm{m}, 10 \mu \mathrm{m}, 5 \mu \mathrm{m}, 2.5 \mu \mathrm{m}$, and $500 \mathrm{~nm}$ on the VACNT film, which were all initially $608 \pm 20 \mu \mathrm{m}$ thick.

The effects of different laser processing parameters on the carbon nanotubes had been explored, including Raman spectroscopy as a function of laser power, which were also were characterized by X-ray photoelectron spectroscopy (XPS) to explore the impacts of carbon ablation on the VACNTs chemical composition and crystallography before and after processing. 


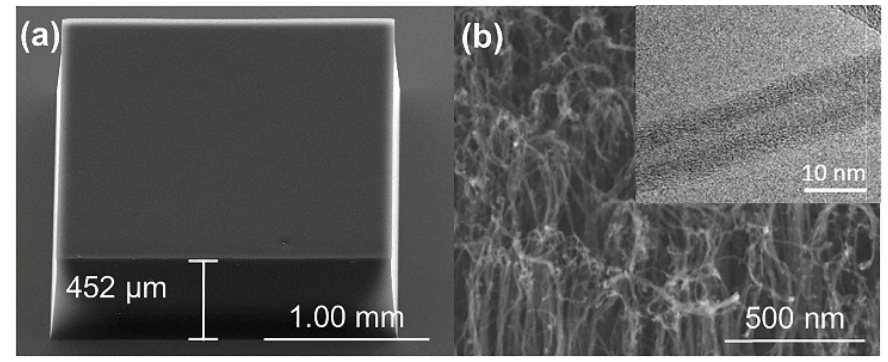

(d)

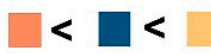

Distance from focus

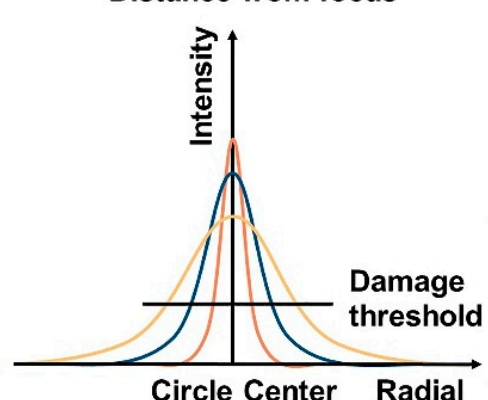

Circle Center Radial (e)

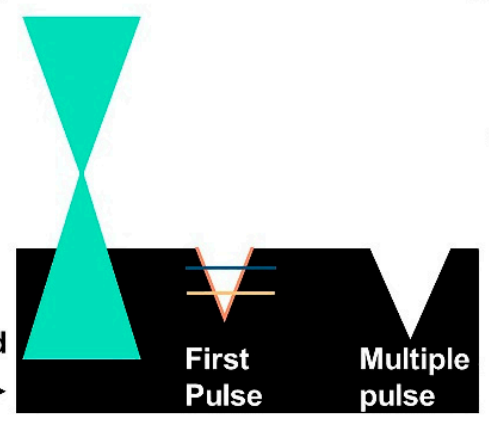

(c)

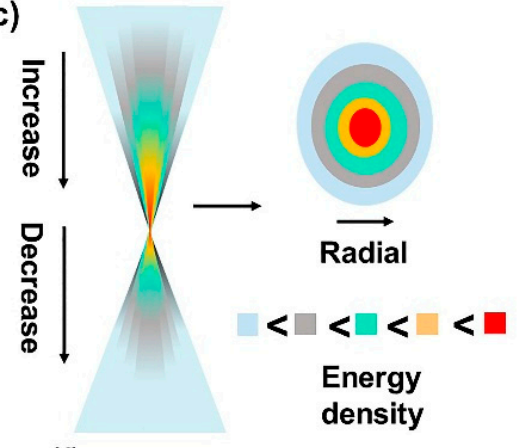

(f)

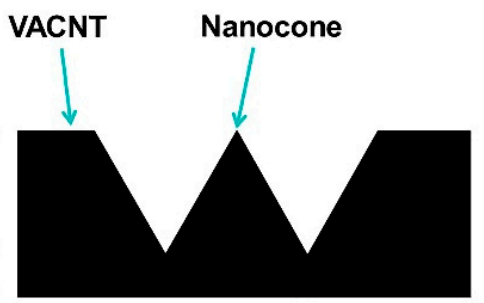

(g)

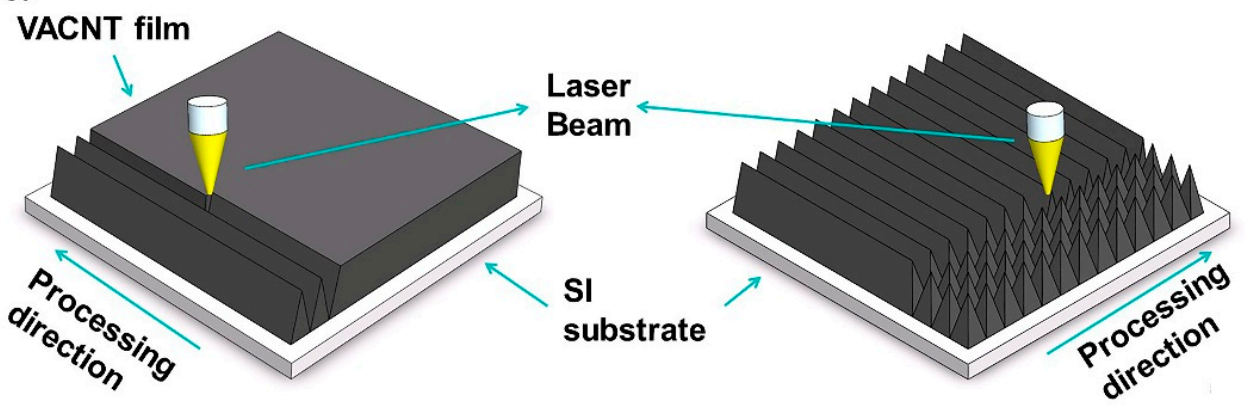

Figure 1. Schematic of the VACNT UV laser patterning process: (a) SEM images of a typical as-synthesized PECVD VACNT thin film (scale bar: $1 \mathrm{~mm}$, Tilt: $45^{\circ}$ ). (b) High-resolution SEM image of the VACNT thin film upper surface highlighting the surface disorder and residual catalyst particles (scale bar: $500 \mathrm{~nm}$, Tilt: 45 ${ }^{\circ}$ ). Inset: High-resolution TEM of an individual as-grown PECVD CNT showing a wall thickness of approximately $0.12 \mathrm{~nm}$, consisting of three graphitic side walls (scale bar: $10 \mathrm{~nm}$ ). (c) Energy distribution diagram of the laser beam. (d) Gaussian distribution of laser beam energy. (e) The process of laser beam energy acting on VACNT to form its shape. (f) Nanocone and (g) Nanocone array formation process.

Field-emission measurements were carried out in a custom-built vacuum chamber evacuated to a base pressure of $<1 \times 10^{-7}$ mbar. Measurements were conducted in diode mode. To minimize anode-induced arcing, the anode was formed from a $5 \mathrm{~mm}$ thick mechanically polished stainless-steel plate (surface roughness), with the cathode formed from either a processed or unprocessed VACNT thin film on the stainless-steel substrate, in which the distance between cathode and anode was $462 \pm 20 \mu \mathrm{m}$. Voltages were swept from 0 to $10 \mathrm{kV}$ with $\Delta \mathrm{V}=50 \mathrm{~V}$ and a dwell/step time of $1 \mathrm{~s}$. A schematic diagram of the test equipment is in Supplementary Materials Figure S3.

A three-dimensional numerical calculation by Comsol Multiphysics software (Version 5.5, Comsol, Inc., Stockholm, Sweden) was carried out to verify the influence of field screen effect on carbon nanotubes cold cathode. (See Supplementary Materials for details of the simulation). 


\section{Results and Discussion}

\subsection{Laser Processing}

In the present work, the nanosecond UV laser optical ablation system is employed, which represents a cheaper and more widely available means of accessing carbon ablation whilst benefitting from a known strong leading UV absorption edge within graphitic carbon nanomaterials $[13,14,22]$. This will be more conducive to the realization of industrialized mass production. Compared with infrared laser processing methods that use the thermal effects to burn out the VACNT [10,23,24], the high-energy ultraviolet photons are more likely to directly destroy the molecular bonds of the material and make the molecules separate from the original material [10]. In addition, the UV laser can achieve a smaller spot and smaller heat-affected zone, which means that it can achieve more sharp machining. The most important parameters in the processing include the lasing frequency $(20-150 \mathrm{kHz})$, optical power $(0-12 \mathrm{~W})$, scanning speed $(0.01-10,000 \mathrm{~mm} / \mathrm{s})$, scanning times, and the distance between the sample and the focus (the Supplementary Materials Figures S4 and S5 shows more details of laser machining).

Figure 1 shows the formation mechanism of the NCNA. As shown in Figure 1c, the maximum energy density and the minimum spot are obtained at the focus. After passing through the focus, the energy begins to diverge, and the spot slowly becomes larger. However, due to the propagation loss of light in the air, the energy distribution of the two positions symmetrical about the focus is not the same. In the radial direction of the spot, the energy of the spot center is the highest and decreases to 0 along the radial direction. The energy distribution law of the laser conforms to the Gaussian distribution, that is, the curve shown in Figure 1d. Therefore, when the laser beam hits the VACNT, the energy impact on the upper surface of the VACNT should conform to the energy distribution corresponding to the spot.

After the first pulse, the CNT in the area where the energy is greater than the damage threshold (ablation threshold of the VACNTs approximately $50 \mathrm{~mJ} \cdot \mathrm{cm}^{-2}$ [25]) of VACNT will be removed, leaving a shape similar to that above the damage threshold of the Gaussian curve on VACNT (it is assumed that the components of energy points in all directions are almost uniform), which can be approximated as a triangle, as shown in Figure 1e. The second pulse will continue to act on VACNT along the shape generated by the first pulse. Since the laser beam energy has the characteristics of Gaussian distribution, at this time, the energy impact on each point on the edge of the shape formed by the first pulse will follow different Gaussian distribution curves. The formation of the final shape of the groove processed on VACNT is the result of the accumulation of multiple energy points with Gaussian distribution following different characteristics in space. According to our research on laser processing parameters (Supplementary Materials) and the previous reports of Tang [20] et al., after multiple pulses, the shapes of grooves processed on VACNT are not the same, one of which is triangular. The nanoscale edges can be obtained by accurately controlling the position of two adjacent triangular machining grooves, as shown in Figure 1f. Thus, NCNA can be obtained by continuous transverse and longitudinal scanning of VACNT, as shown in Figure $1 \mathrm{~g}$.

According to the above discussion, the change of focus value will make the upper surface of VACNT in Gaussian energy distribution have different characteristics, which will change the width and depth of the area that can be damaged by the first pulse energy and then affect the shape of the machining groove. The increase (decrease) of power will not change the energy distribution characteristics of the upper surface of VACNT but will increase (decrease) the energy as a whole; that is, in the same Gaussian distribution characteristics, a wider (narrower) area will reach the damage threshold of VACNT, thus affecting the length and width (or the size and shape of the area affected by each pulse) of the machining groove. The frequency mainly changes the number of pulses per unit time. Together with the scanning speed and scanning times, it controls the number of pulses in the unit area. The scanning speed and scanning times jointly control the residence time of the laser beam on the VACNT. The difference is that if the scanning times are 
fixed, adjusting the laser beam residence time by adjusting the scanning speed means that the thermal effect of the laser on the VACNT is more continuous. On the contrary, if the scanning speed is fixed, adjusting the scanning times may reduce the accumulation of thermal effects in a certain area. To some extent, laser frequency, scanning speed, and scanning times also affect the shape of the ablated groove. Of course, their cooperation is particularly important.

\subsection{Impacts on the Structure and Surface Pre-and Post-Processing}

To further explore the chemical and physical impacts of the UV laser ablation on the processed VACNT, Raman spectra and XPS were undertaken pre and post-processing. Figure $2 \mathrm{a}$ shows the VACNT with only the right half processed. Positions 1, 2, and 3 correspond to the processing position, the junction of processing and unprocessed position, and the unprocessed position, respectively. The Raman test results at positions 1, 2, and 3 have been shown in Figure $2 b$. The D peak was mainly induced by structural defects of CNT, amorphous carbon, or contaminants, and the G peak represents the degree of SP2 hybridization in CNT [26]. From position 1-3, the intensity of the D peak increased gradually and the G peak decreased gradually, with the ID/IG values of positions 1, 2, and 3 being $0.93,0.70$, and 0.48 . It indicated that those positions (position 1 ) processed had more structural defects than the unprocessed sites (position 3). During processing, high-energy ultraviolet photons might directly degrade the chemical bonds within carbon nanotubes; at the same time, the heat generated during processing may also destroy the chemical bond and form pyrolytic carbon, broken chemical bonds combined with other elements in the air, resulting in more defects and amorphous carbon [27]. Position 2 is less affected than position 1, so its ratio of ID to IG is slightly less than that of position 1 . We also tested the ID/IG values of groove edges with different powers; they were all higher than the unprocessed position. This may be because in the process of UV laser processing, the number of defects caused by photon direct cutting bonding is greater than that caused by the thermal ablation effect, which makes the value of ID/IG near the ablation groove higher (see Supplementary Materials Figures S6 and S7).

Interestingly, we found some nanoparticles at the top of the processed CNTs and the junction of the processed and the unprocessed regions, as shown in Figure 2c. This is likely residual Fe and $\mathrm{Al}$ catalyst materials, which both, when optically excited, reacted with the VACNTs and the ambient gaseous environment to form new large exotic nanoparticles [28]. The optical coupling to these metallic nanoparticles likely enhanced the optical coupling to the VACNT system, allowing us to access low-power densities. This shows that there is a thermal effect in the UV laser processing of carbon nanotubes. In some previous reports using femtosecond laser [19], the ablated edge has fewer defects than unprocessed. It has been shown elsewhere that temperatures of up to $1000{ }^{\circ} \mathrm{C}$ can be effective at driving the graphitization of otherwise defective nanocarbons due to their small size [29]. However, there are also some findings of Hai et al. [23] which have shown that elevated temperatures over $525^{\circ} \mathrm{C}$ can result in the combustion of carbon nanotubes that further lead to material removal due to $\mathrm{CO}$ and $\mathrm{CO}_{2}$ formation. It was proposed that the high temperature generated in the laser processing helps to burn amorphous carbons away [30].

XPS showed that in addition to $\mathrm{C}$, there is a small amount of $\mathrm{O}$ in the raw VACNT. Compared with the raw VACNT, the O concentration in the UV laser-processed VACNT was significantly increased, and there is a small amount of $\mathrm{N}$ and other trace elements in the air. The $\mathrm{C} 1 \mathrm{~s}, \mathrm{O} 1 \mathrm{~s}$, and $\mathrm{N}$ 1s peaks of processed VACNT and the $\mathrm{C} 1 \mathrm{~s}$ and $\mathrm{O} 1 \mathrm{~s}$ peaks of unprocessed VACNT are clearly visible in the XPS survey scan spectrum (see Supplementary Materials Figure S8a). The O concentration in the raw VACNT is 0.97\%; the $\mathrm{O}$ and $\mathrm{N}$ concentrations in the processed VACNT are $7.54 \%$ and $0.88 \%$. We examined the $\mathrm{C}$ 1s XPS peaks for processed (Figure 2d) and raw VACNT (see Supplementary Materials Figure $\mathrm{S} 8 \mathrm{~b}$ ). As shown in Figure $2 \mathrm{~d}$, peak I represents the graphite-like $\mathrm{C}-\mathrm{C}$ bonds at $284.4 \mathrm{eV}$, the peaks at $285.3 \mathrm{eV}$ (II) and $286.4 \mathrm{eV}$ (III) are the different types of the C-N bones, peak II corresponds to the SP2 trigonal C-N bonding, and peak III corresponds 
to SP3 tetrahedral C-N bonding, while the last peak (IV) at $289.3 \mathrm{eV}$ is attributed to $\mathrm{C}-\mathrm{O}$ type bonds [31-34]. The $\mathrm{C}$ 1s peaks of the raw VACNT only show graphite-like $\mathrm{C}-\mathrm{C}$ bonds and $\mathrm{C}-\mathrm{O}$ type bonds. $\mathrm{O}$ in the unprocessed samples may be due to the oxidation of the VACNTs during post-growth air exposure [32].
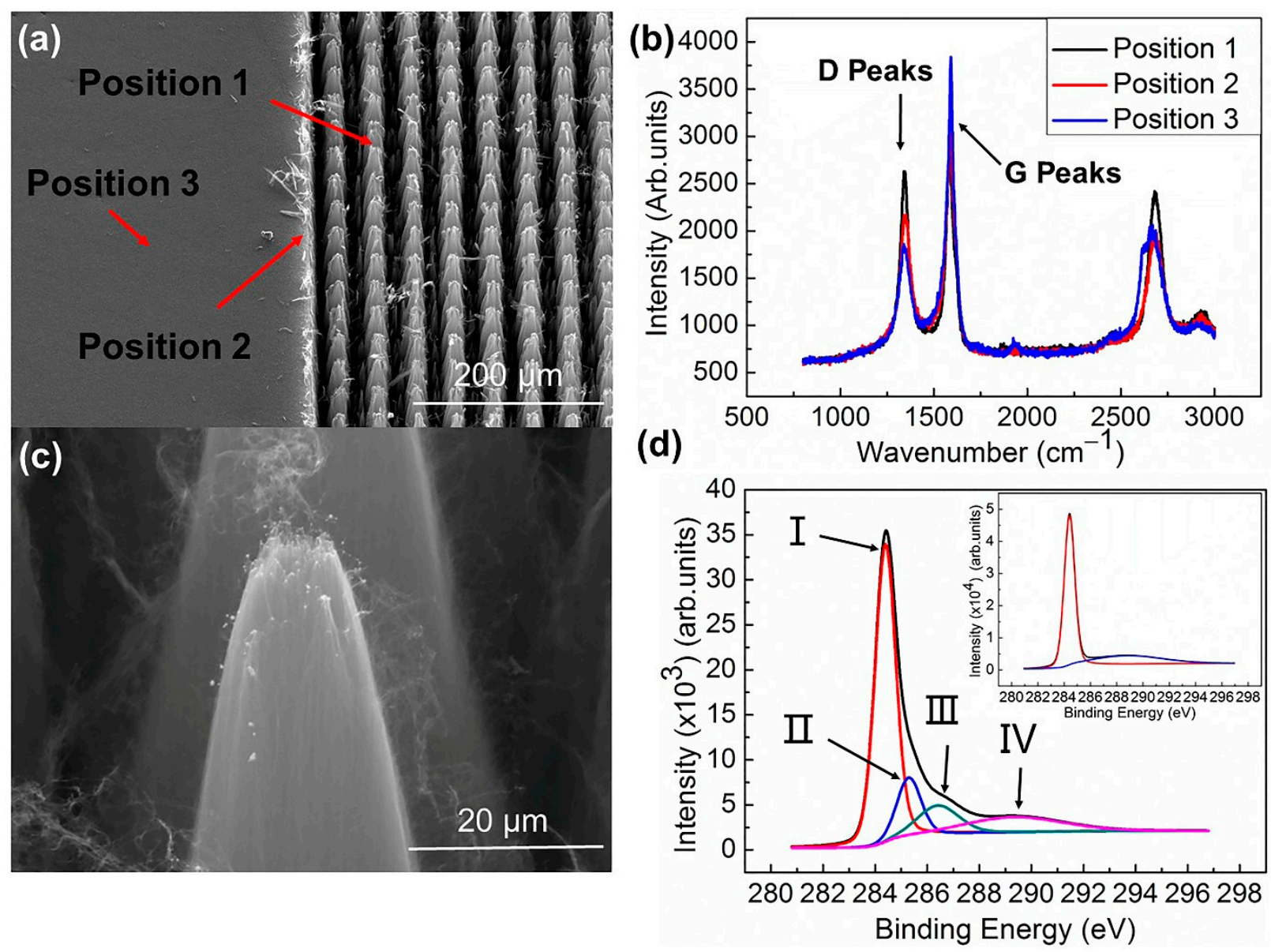

Figure 2. (a) A typical SEM image of a patterned VACNT sample where the right side has been processed. Position 1 is the top of the laser processed array, position 2 is the junction of the processed position and the unprocessed position, and position 3 is the unprocessed position. (b) Corresponding Raman spectra at the positions 1, 2, and 3. (c) New large exotic nanoparticles of $\mathrm{Al}$ and Fe on the processed VACNT. (d) The C 1s XPS peaks for processed VACNT.

The appearance of $\mathrm{N}$ and other trace elements, as well as the increase in $\mathrm{O}$ content in the samples after UV laser processing is likely due to reactions with some elements in the air combining with $C$ atoms with incomplete chemical bonds after the UV photons cut off the chemical bonds and finally doped into VACNT to form some vacancy-related defects. In terms of electronic state, the presence of surface-localized oxygen molecules has played a positive role in improving the field emission performance due to the generation of new states induced by the $\mathrm{O}_{2}$ [35]. $\mathrm{N}$ doping is commonly beneficial; it enhances electron emission because doped nitrogen atoms could replace carbon atoms in carbon nanotubes and therefore increase the electronic density [35]. In terms of structure, the $\mathrm{O}$ and $\mathrm{N}$ all had been shown to improve electron emission because they can usually make carbon nanotubes form open edges, and also the engineered tip morphologies provide more small emission tips on the surface of carbon nanotubes [31,36,37]. Our findings suggest that the $5 \%$ composition of the identified $\mathrm{N}$ and $\mathrm{O}$ tends to vary between samples, which is likely due to the energy sensitivity and inevitable process variability of the bond formation (XPS survey scan spectrum and the C1s XPS peaks of another sample showed in Supplementary Materials Figure S8c,d). 


\subsection{Field Emission Properties as a Function of Conical Tip Radius of Curvature}

To study the influence of the processed VACNT tip radius of curvature on their field emission properties, cuboid arrays with a side length of about $20 \mu \mathrm{m}$ and spacing of about $40 \mu \mathrm{m}$ were firstly fabricated and UV laser processed to create tips of the engineered radius of curvature of $20 \mu \mathrm{m}, 10 \mu \mathrm{m}, 5 \mu \mathrm{m}, 2.5 \mu \mathrm{m}$, and $500 \mathrm{~nm}$. Figure $3 \mathrm{a}-\mathrm{d}$ shows the morphology arrays with a side length of about $20 \mu \mathrm{m}$ and $500 \mathrm{~nm}$; Figure $3 \mathrm{e}, \mathrm{f}$ are the enlarged view of their local area and show one of the arrays. Photographs of other tip sizes and some typical samples are included in the Supplementary Materials Figure S9.

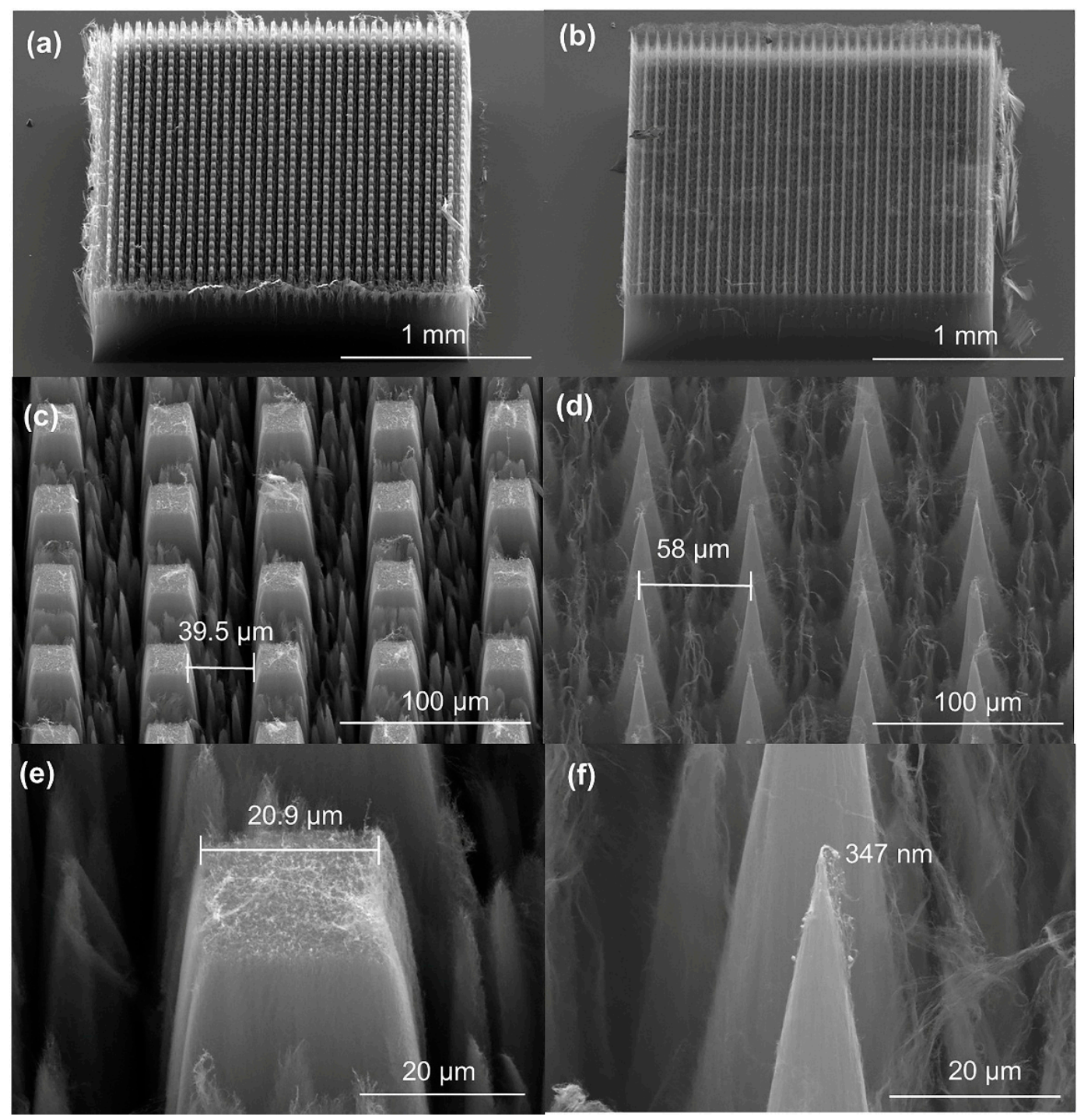

Figure 3. (a) A typical UV laser-processed cuboid array with a side length of about $20 \mu \mathrm{m}$ and (b) $500 \mathrm{~nm}$ consisting of 1089 elements. (c) An enlarged view of the processed array showing the formed tips of about $20 \mu \mathrm{m}$ and (d) about $500 \mathrm{~nm}$. (e) A single tip in the array with a radius of curvature of $20.9 \mu \mathrm{m}$ and (f) $347 \mathrm{~nm}$.

Due to the slight jitter of the carbon nanotube film fixed on the sample table by vacuum suction during the processing, the shape and size of each small array may be slightly different, which is within the allowable error range. Our electron microscopy studies highlighted several small ( $<50 \mathrm{~nm}$ diameter) CNT emitters within the processed arrays, which we believe positively contribute to the enhanced electron emission. Further work is ongoing to investigate the kinetics associated with their formation so as to derive further control in their placement and formation. Meanwhile, the emitters of some small carbon nanotubes are relatively chemically stable. (Please refer to Supplementary Materials Figure S10 for photos). 
To examine the impact of tip radius of curvature on the field electron emission performances, unprocessed and processed VACNT film cathodes were tested in a custom-build ultra-high vacuum environment; all tested samples had a similar height $(608 \pm 20 \mu \mathrm{m})$ and the same number of pillars. Figure 4 a shows the current density as a function of the applied global electric field for a representative raw sample and cone arrays of tip sizes $500 \mathrm{~nm}, 5 \mu \mathrm{m}$, and $15 \mu \mathrm{m}$. The nanocone sample with a tip size of $500 \mathrm{~nm}$ had the best performance. Its turn-on electric field (defined as reported elsewhere, specifically as the electric field that stimulates an emission current of $1 \mu \mathrm{A}$ ) is $1.6 \mathrm{~V} / \mu \mathrm{m}$, which decreased by $1 \mathrm{~V} / \mu \mathrm{m}$. Its emission current density reached up to $144.5 \mathrm{~mA} / \mathrm{cm}^{2}$ (the calculated emission area of the $500 \mathrm{~nm}$ nanocone array is $4 \mathrm{~mm}^{2}$ ) at the applied electric field of $3.2 \mathrm{~V} / \mu \mathrm{m}$. At the applied field of $3.2 \mathrm{~V} / \mu \mathrm{m}$, the current density is enhanced by 97 times compared with the unprocessed sample. If only considering the area of the tips, the current density of the nanocone array with a tip size of $500 \mathrm{~nm}$ is $2.703 \times 10^{6} \mathrm{~mA} / \mathrm{cm}^{2}$ (the calculated emission area of $500 \mathrm{~nm}$ nanocone array is $213.8 \mu \mathrm{m}^{2}$ ), which are $1.814 \times 10^{6}$ times higher than that of the raw sample. More than three samples for each tip diameter were tested, and the data were plotted in the J- $\mathrm{E}_{\text {on }}$ plot in Figure $4 \mathrm{~b}$. The observed variation in the measured performance of the processed emitters is due to the relative youthfulness of the ablation patterning process, which induces observed marginal morphological sample-to-sample variations. As FE is particularly sensitive to surface chemistry as well as emitter geometry, such small variations result in somewhat larger observed variations in the J-E $\mathrm{E}_{\text {on }}$ data. In what follows, we take the average value for each tip set of cone array for comparison.

(a)
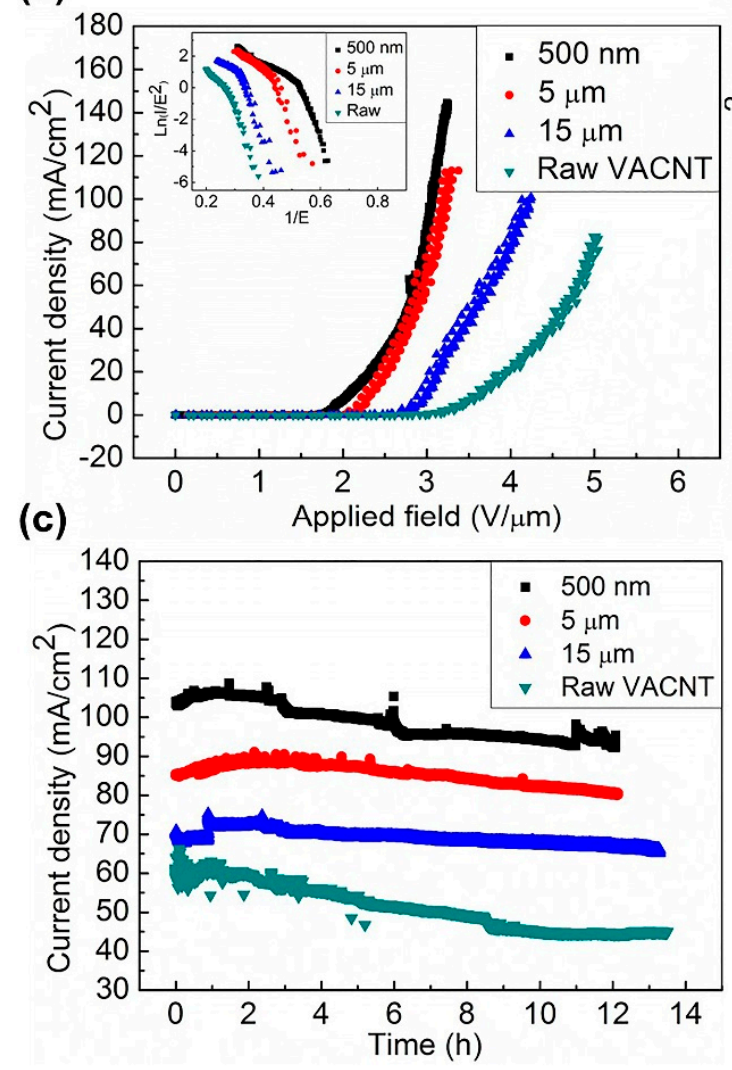

(b)
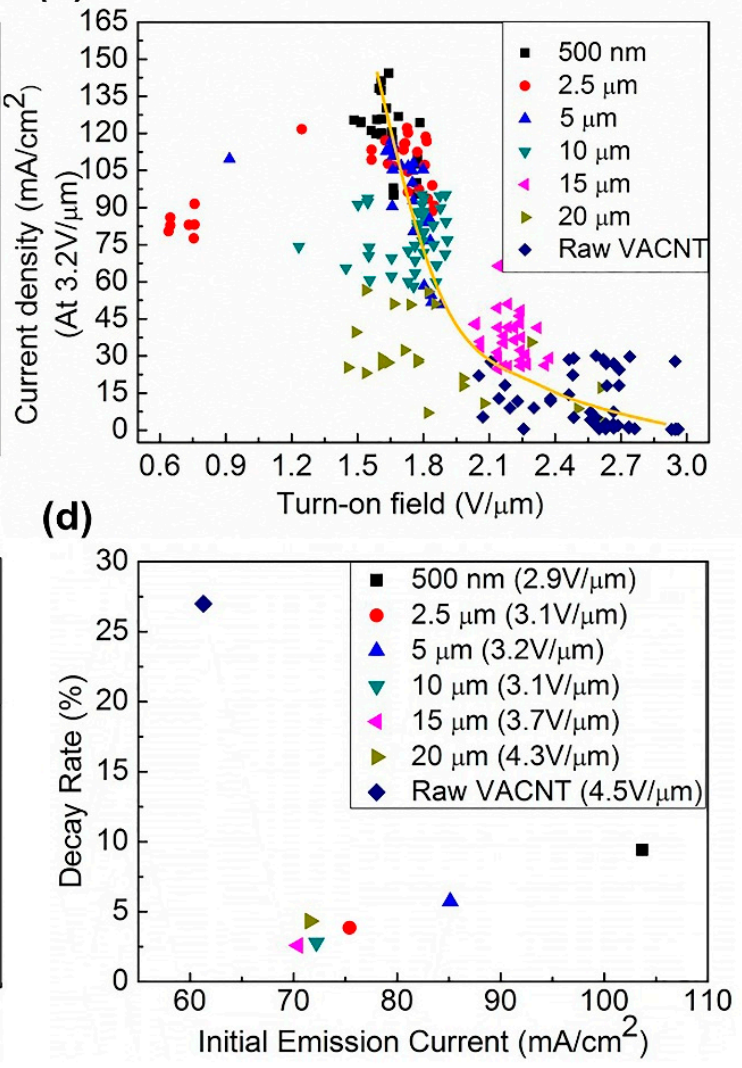

Figure 4. (a) Current density-applied field curves of the raw VACNT film; the cone arrays of the tip size are about 500 nm, $5 \mu \mathrm{m}$, and $15 \mu \mathrm{m}$, and the illustration is Forwer-Nordheim plots. (b) The statistical diagram of the relationship between the open field strength and the current density of the unprocessed samples and the cone array samples with different tip sizes. (c) Current-time stability curves of the raw VACNT film; the cone arrays of the tip size are $500 \mathrm{~nm}, 5 \mu \mathrm{m}$, and $15 \mu \mathrm{m}$. (d) The relationship between the current decay rate $(12 \mathrm{~h})$ and the initial current of raw samples and cone array samples with different tip sizes was studied. (The calculated emission is $4 \mathrm{~mm}^{2}$ from (a-d)). 
Our post-emission SEM findings suggested that laser processing tended to marginally compromise the degree of adhesion of the VACNTs with the underlying substrate. Figure $4 \mathrm{c}$ shows the DC temporal stability, conducted over $12 \mathrm{~h}$, of these VACNT samples. Compared with the unpatterned samples, nanocone-patterned samples were noted to be more stable. Figure $4 \mathrm{~d}$ shows the relationship between the initial current and the decay rate of raw VACNT and cone arrays with different tip sizes. It can be seen that the UV laser-processed arrays generally have higher stability and simultaneously achieve higher total current and lower current decay. We also found under the same experimental conditions that the field emission impact damage observed on the samples of the tested UV laser-processed samples was significantly smaller than that of the unprocessed samples, which was due to the lower driving voltage. (See Supplementary Materials Figure S11 for pre and post-field emission material characterization).

\subsection{Simulation of Electric Field Intensity with a Different Tip Radius of Curvature}

A three-dimensional numerical calculation by Comsol Multiphysics software was carried out. According to the calculation results of the three models, the screen effect of the nanocone array with a tip size of $500 \mathrm{~nm}$ is the weakest (Figure 5c), which is followed by the arrays of tip size of $20 \mu \mathrm{m}$ (Figure $5 \mathrm{~b}$ ), and the strongest screening effect is the raw VACNT film (Figure 5a). The nanocone arrays that have a tip size of $500 \mathrm{~nm}$ have more regions with higher electric field intensity, which are followed by the arrays with a tip size of $20 \mu \mathrm{m}$. According to the actual situation, each carbon nanotube has a certain field screen effect on the surrounding carbon nanotubes, and the strength of the field screen effect is related to their height and the distance between them [38]. The grooves formed by laser processing of the cone arrays with tip sizes of $500 \mathrm{~nm}$ and $20 \mu \mathrm{m}$ help to reduce the field screen effect and make the edge and tip carbon nanotubes obtain higher electric field strength. Moreover, the sharp area is more conducive to the increase in electric field strength. Figure $5 \mathrm{~d}$ shows the calculation results of the electric field strength at positions 1 (the center point of the upper surface of the center array corresponds to the center point of the upper surface of VACNT), 2 (the center point of the upper surface of the third array of edges corresponds to the position of the same coordinates of VACNT), and 3 (the center point of the upper surface of the corner array corresponds to the same coordinate position of VACNT) of the cone arrays of tip size from 0.5 to $20 \mu \mathrm{m}$ and the raw VACNT. It shows the increasing trend of the electric field at positions 1,2, and 3 with the decrease in tip size and their comparison with unprocessed VACNT. It also shows the comparison results at different positions (positions 1, 2, and 3).

The processed sample model has higher field strength at positions 1, 2, and 3, and the field strength tends to increase with the decrease in tip size. At the same time, the field strength at different positions in the same model is also different. Compared with the center position, the number of adjacent carbon nanotubes at the corner position is less and the shielding effect is smaller, so it has higher field strength. However, in the case of the same area, the smaller diameter means that the number of carbon nanotubes is less, which means that the number of emitters is also reduced. The game between the two is the two main factors that determine the field emission performance. 


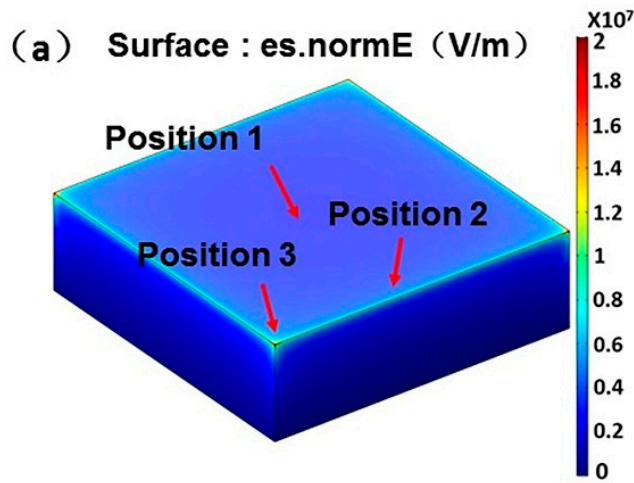

(c) Surface : es.normE (V/m)

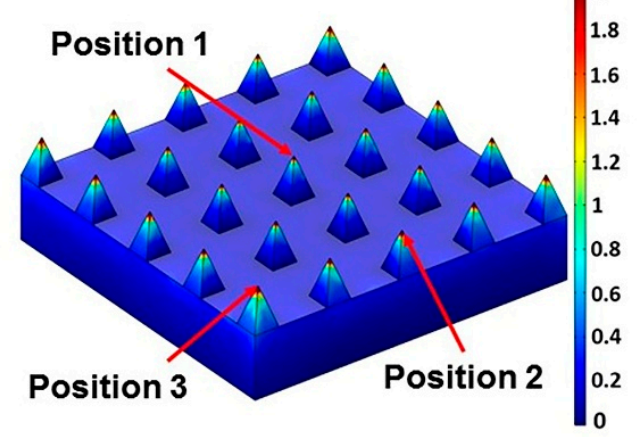

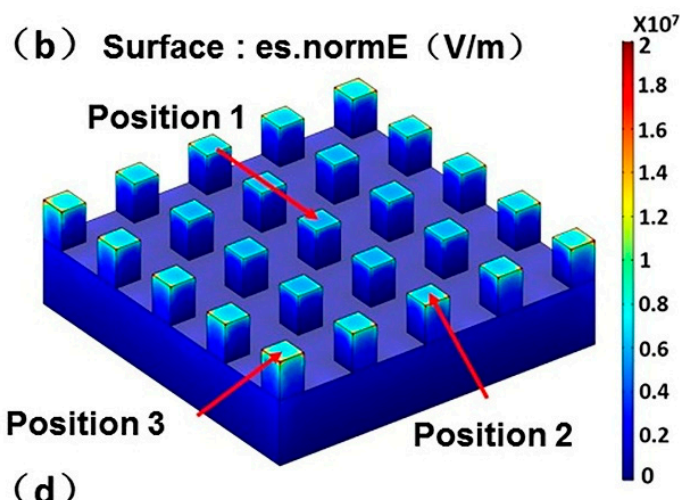

(d)

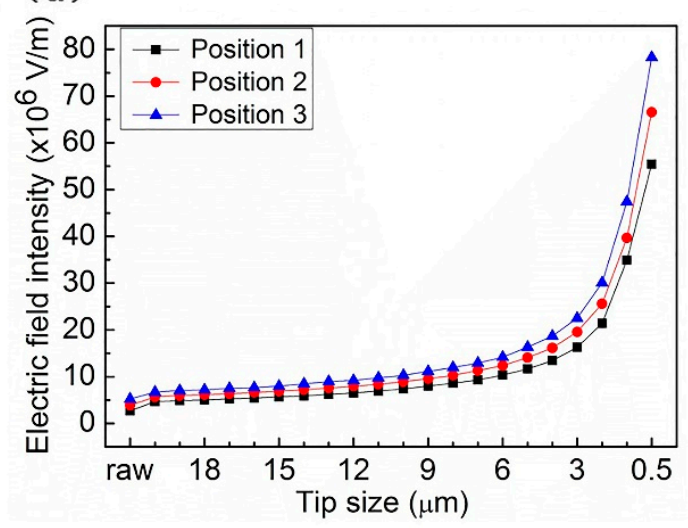

Figure 5. (a) The electric field strength distribution of the model of raw VACNT film. (b) The electric field strength distribution of the model of the arrays with a tip size of $20 \mu \mathrm{m}$. (c) The electric field strength distribution of the model of the nanocone arrays with a tip size of $500 \mathrm{~nm}$. (d) The electric field strength calculation results of the middle point (Position 1, the center point of the upper surface of the center array corresponds to the center point of the upper surface of VACNT), edge-point (Position 2, the center point of the upper surface of the third array of edges corresponds to the position of the same coordinates of VACNT), and the center point (Position 3, the center point of the upper surface of the corner array corresponds to the same coordinate position of VACNT) of the top surface of the cone arrays of tip size from $0.5-20 \mu \mathrm{m}$ and the raw VACNT.

\section{Results and Discussion}

Here, we report a method of patterning VACNT thin film via inexpensive and universal ultraviolet laser ablation. Through the study of the interaction process between laser and VACNT and the reasonable control of this process, the nanocone arrays are fabricated by using a micron light spot. Then, the impacts of this facile patterning were explored with regard to the CNT metrology and the field electron emission properties. The maximum turn-on electric field of a CNT cold cathode has been shown to reduce by over $1 \mathrm{~V} / \mu \mathrm{m}$ as a result of UV laser patterning, and the maximum current density increasing by more than $97 \times$ (average current of overall area). The $12 \mathrm{~h}$ stability test shows that the maximum reduction of the current attenuation rate is more than $20 \%$, with the VACNT samples proving to be increasingly robust and less easily damaged by the strong electric fields during emission. The results of Raman spectroscopy and XPS show that the UV laser-processed VACNTs have more structural defects as well as increased trace elements leading to likely doping during the UV laser processing, which we attribute to the in-air operation of the system. This may be due to the ablation of carbon nanotubes by removing the thermal effect in processing, and high-energy photons tend to directly break the chemical bonds of carbon nanotubes. Some fine carbon nanotube emitters formed by processing may also be helpful for emission. Numerical simulations validated our empirical findings, evidencing that the surface field strength of the processed array can be enhanced by more than one 
order of magnitude under the same conditions, the field enhancement factor is greatly improved, and the shielding effect is greatly reduced.

In general, this method can easily manufacture a cold cathode electron source with high and stable current density and low turn-on electric field on a large scale at a low cost. It is hoped that the presented facile and inexpensive laser patterning strategy will open up new processing opportunities for emerging nanomaterials-based technologies and in doing so will accelerate the adoption and integration of emerging $1 \mathrm{D}$ and $2 \mathrm{D}$ nanomaterials in a range of new applications.

Supplementary Materials: The following are available online at https://www.mdpi.com/article/ 10.3390/nano11123244/s1, Figure S1: Large aspect ratio array processed by the UV laser. (a) The aspect ratio exceeds 12. (b) The aspect ratio is about 14 (Tilt: $45^{\circ}$ ), Figure S2: (a) CAD drawings for machining and (b) corresponding processed shape, Figure S3: The simple schematic diagram of field emission test device, Figure S4: (a) For VACNT processed with different frequencies, the number in the figure is the frequency value used $(\mathrm{kHz})(20 \%$ maximum output power, scanning once, scanning speed $200 \mathrm{~mm} / \mathrm{s}$, focusing distance $-300 \mu \mathrm{m}$ ), which means that based on the characteristics of the laser, the pulse width and maximum output power will change correspondingly with the change of frequency, (b) is the curve of maximum output power and pulse width with frequency, Figure S5: SEM images of the effect of: (a) Different power (Focal distance: $-150 \pm 100 \mu \mathrm{m}$, scanning times: $1 \mathrm{~s}$, scanning speed: $200 \mathrm{~mm} / \mathrm{s}$ ). (b) Different defocus distance (A positive value is a positive focus and a negative value is a negative focus, in units of $\mu \mathrm{m}$. power: $2.3 \mathrm{~W}$, scanning times: 1 , scanning speed: $200 \mathrm{~mm} / \mathrm{s}$ ) (c) Different scanning times (power: $1.9 \mathrm{~W}$, defocus distance: $-150 \pm 100 \mu \mathrm{m}$, scanning speed: $200 \mathrm{~mm} / \mathrm{s}$. Right illustration parameters: frequency: $80 \mathrm{kHz}$, pulse width: $18.5 \mathrm{~ns}$, power: $2.2 \mathrm{~W}$, speed: $150 \mathrm{~mm} / \mathrm{s}$ ) (d) Different scanning speed (in units of $\mathrm{mm} / \mathrm{s}$, power: $2.03 \mathrm{~W}$, Defocus distance: $-150 \pm 100 \mu \mathrm{m}$, scanning speed: $200 \mathrm{~mm} / \mathrm{s}$ ), Figure S6: Original Raman spectra of the raw area and A-O points marked in Figure S4a. In all Raman measurements in this paper, the Raman laser spot is smaller than the tip size of the measured array. (a) Original Raman spectra of the point A marked in Figure S4a. (b) Original Raman spectra of the point B marked in Figure S4a. (c) Original Raman spectra of the point C marked in Figure S4a. (d) Original Raman spectra of the point D marked in Figure S4a. (e) Original Raman spectra of the point E marked in Figure S4a. (f) Original Raman spectra of the point F marked in Figure S4a. (g) Original Raman spectra of the point G marked in Figure S4a. (h) Original Raman spectra of the point H marked in Figure S4a. (i) Original Raman spectra of the point I marked in Figure S4a. (j) Original Raman spectra of the point J marked in Figure S4a. (k) Original Raman spectra of the point K marked in Figure S4a. (l) Original Raman spectra of the point L marked in Figure S4a. (m) Original Raman spectra of the point M marked in Figure S4a. (n) Original Raman spectra of the point N marked in Figure S4a. (o) Original Raman spectra of the point O marked in Figure S4a. (p) Original Raman spectra of the raw area, Figure S7: The ID/IG value of the Raman spectrum of the raw and the point $\mathrm{A}$ to point $\mathrm{O}$ marked in Figure S4a, Figure S8: (a) The XPS survey scan spectrum and (b) the C1s XPS peaks of the raw sample. (c) The XPS survey scan spectrum and (d) The C1s XPS peaks of another sample (The content ratio of main elements is C:O:N:S = 89.437:9.958:0.455:0.15), Figure S9: The typical picture of other nanocone arrays which have different tip sizes processed by the UV laser. (a-c) The tip size is approximately $2.5 \mu \mathrm{m}$. (d-h) The tip size is approximately $5 \mu \mathrm{m}$. (i-k) The tip size is approximately $10 \mu \mathrm{m}$. (l-n) The tip size is approximately $15 \mu \mathrm{m}$, Figure S10: Fine carbon nanotubes on an array processed by the UV laser. (a) Fine emitters on conical arrays and (b) Enlarged view of fine emitter on the side. (c) Fine emitters with more uniform orientation on the rectangular cylinder array and (d) Enlarged view of fine emitter on the side. (e) An array of rectangular cylinders with only small emitters on the side and (f) Enlarged view of fine emitter on the side, Figure S11: Comparison of the morphology of processed and unprocessed samples tested under the same conditions. (a) The raw VACNT before tested. $(\mathrm{b}-\mathrm{c})$ The processed Cone array and $(\mathrm{d}-\mathrm{e})$ Cone cylinder array with the tips before tested. $(\mathrm{f}-\mathrm{i})$ The VACNT after tested, a large area of VACNT was damaged and deep holes appeared during the test. $(j-m)$ The processed cone array after being tested, A small area was damaged during the test, mainly at the edge, and a small part of the top of the array showed the explosive fracture. $(n-q)$ Cone array with tip after tested. Only a few arrays on the edge were damaged during the test. A few arrays changed slightly during the test. 
Author Contributions: According to the journal proposed scheme, the authors have contributed in the following ways: Conceptualization: C.L.; Methodology: C.L. and J.Z.; Software and Simulation: J.Z., X.G., W.L., H.T., Y.Q. and K.C.; Validation: C.L., M.T.C., Y.L. and Z.L.; Formal analysis: J.Z., Y.L. and X.L.; Investigation: J.Z., Y.L. and J.X.; Resources: C.L., Y.L., Z.L. and Q.D.; Data curation: J.Z., S.Z., J.X. and A.W.; Writing—original draft preparation: J.Z.; Writing-review and editing: C.L., M.T.C. and Q.D.; Visualization: J.Z. and G.L.; Supervision: C.L., Z.L., Y.L., A.W. and Q.D.; Project administration: C.L., Y.L. and Q.D.; Funding acquisition: C.L. and Q.D. All authors have read and agreed to the published version of the manuscript.

Funding: This work is supported by the Strategic Priority Research Program of Chinese Academy of Sciences (Grant No. XDB36000000), the National Natural Science Foundation of China (Grant No. 51972072, 52072084, 51925203), CAS Interdisciplinary Innovation Team (Grant No. JCTD-2018-03), the Key Area Research and Development Program of Guangdong Province (Grant No. 2020B0101020002), the GBA National Institute for Nanotechnology Innovation (Grant No. 2020GN0106), and the National Key R\&D Program of China (Grant No. 2016YFA0202000).

Institutional Review Board Statement: Not applicable.

Informed Consent Statement: Not applicable.

Data Availability Statement: The data presented in this study are available on request from the corresponding author.

Conflicts of Interest: The authors declare no conflict of interest. The funders had no role in the design of the study, in the collection, analyses, or interpretation of data, in the writing of the manuscript; or in the decision to publish the results.

\section{References}

1. Liang, B.; Zhang, Z.; Chen, W.; Lu, D.; Yang, L.; Yang, R.; Zhu, H.; Tang, Z.; Gui, X. Direct Patterning of Carbon Nanotube via Stamp Contact Printing Process for Stretchable and Sensitive Sensing Devices. Nanomicro Lett. 2019, 11, 92. [CrossRef] [PubMed]

2. Lee, M.W.; Lai, W.C.; Kao, Y.Y.; Hsieh, T.L.; Huang, K.J. Angle-Resolved Field Emission of Individual Carbon Nanotubes. Jpn. J. Appl. Phys. 2009, 48, 072402. [CrossRef]

3. Li, C.; Li, Z.; Ke, C.; Bing, B.; Qing, D. Edge effect enhanced photo-thermionic emission from a carbon nanotubes array. Appl. Phys. Lett. 2017, 110, 093105. [CrossRef]

4. Li, Z.J.; Yang, X.X.; He, F.; Bai, B.; Zhou, H.; Li, C.; Dai, Q. High current field emission from individual non-linear resistor ballasted carbon nanotube cluster array. Carbon 2015, 89, 1-7. [CrossRef]

5. Ohara, J.; Nagakubo, M.; Kawahara, N.; Hattori, T. High aspect ratio etching by infrared laser induced micro bubbles. Proc. IEEE Micr. Elect. 1997, 175-179. [CrossRef]

6. Zweig, A.D.; Deutsch, T.F. Shock-Waves Generated by Confined Xecl Excimer Laser Ablation of Polyimide. Appl. Phys. B Photo 1992, 54, 76-82. [CrossRef]

7. Choo, K.L.; Ogawa, Y.; Kanbargi, G.; Otra, V.; Raff, L.M.; Komanduri, R. Micromachining of silicon by short-pulse laser ablation in air and under water. Mater. Sci. Eng. A Struct. 2004, 372, 145-162. [CrossRef]

8. Geiger, M.; Becker, W.; Rebhan, T.; Hutfless, J.; Lutz, N. Increase of efficiency for the XeCl excimer laser ablation of ceramics. Appl. Surf. Sci. 1996, 96-98, 309-315. [CrossRef]

9. Geiger, M.; Roth, S.; Becker, W. Microstructuring and surface modification by excimer laser machining under thin liquid films. In Proceedings of the Alt '97 International Conference on Laser Surface Processing, Limoges, France, 8-12 September 1997; Volume 3404, pp. 200-208. [CrossRef]

10. Meijer, J. Laser beam machining (LBM), state of the art and new opportunities. J. Mater. Process. Technol. 2004, $149,2-17$. [CrossRef]

11. Li, C.; Zhou, X.; Zhai, F.; Li, Z.J.; Yao, F.R.; Qiao, R.X.; Chen, K.; Cole, M.T.; Yu, D.P.; Sun, Z.P.; et al. Carbon Nanotubes as an Ultrafast Emitter with a Narrow Energy Spread at Optical Frequency. Adv. Mater. 2017, 29, 1701580. [CrossRef]

12. Li, C.; Chen, K.; Guan, M.; Wang, X.; Zhou, X.; Zhai, F.; Dai, J.; Li, Z.; Sun, Z.; Meng, S.; et al. Extreme nonlinear strong-field photoemission from carbon nanotubes. Nat. Commun. 2019, 10, 4891. [CrossRef]

13. Mizuno, K.; Ishii, J.; Kishida, H.; Hayamizu, Y.; Yasuda, S.; Futaba, D.N.; Yumura, M.; Hata, K. A black body absorber from vertically aligned single-walled carbon nanotubes. Proc. Natl. Acad. Sci. USA 2009, 106, 6044-6047. [CrossRef]

14. Yang, Z.P.; Ci, L.; Bur, J.A.; Lin, S.Y.; Ajayan, P.M. Experimental observation of an extremely dark material made by a low-density nanotube array. Nano Lett. 2008, 8, 446-451. [CrossRef]

15. Elmer, J.W.; Yaglioglu, O.; Schaeffer, R.D.; Kardos, G.; Derkach, O. Direct patterning of vertically aligned carbon nanotube arrays to $20 \mathrm{mu} \mathrm{m}$ pitch using focused laser beam micromachining. Carbon 2012, 50, 4114-4122. [CrossRef]

16. Cole, M.T.; Milne, W.I. Plasma Enhanced Chemical Vapour Deposition of Horizontally Aligned Carbon Nanotubes. Materials 2013, 6, 2262-2273. [CrossRef] 
17. Kumar, A.; Shivareddy, S.G.; Correa, M.; Resto, O.; Choi, Y.; Cole, M.T.; Katiyar, R.S.; Scott, J.F.; Amaratunga, G.A.; Lu, H.; et al. Ferroelectric-carbon nanotube memory devices. Nanotechnology 2012, 23, 165702. [CrossRef] [PubMed]

18. Li, Z.J.; Bai, B.; Li, C.; Dai, Q. Efficient photo-thermionic emission from carbon nanotube arrays. Carbon 2016, 96, 641-646. [CrossRef]

19. Dang, M.N.; Nguyen, M.D.; Hiep, N.K.; Hong, P.N.; Baek, I.H.; Hong, N.T. Improved Field Emission Properties of Carbon Nanostructures by Laser Surface Engineering. Nanomaterials 2020, 10, 1391. [CrossRef] [PubMed]

20. Tang, X.Y.; Yue, H.X.; Liu, L.; Luo, J.; Wu, X.L.; Zheng, R.T.; Cheng, G.A. Vertically Aligned Carbon Nanotube Microbundle Arrays for Field-Emission Applications. ACS Appl. Nano Mater. 2020, 3, 7659-7667. [CrossRef]

21. Cheong, F.C.; Lim, K.Y.; Sow, C.H.; Lin, J.Y.; Ong, C.K. Large area patterned arrays of aligned carbon nanotubes via laser trimming. Nanotechnology 2003, 14, 433-437. [CrossRef]

22. Takagi, Y.; Okada, S. Theoretical calculation for the ultraviolet optical properties of single-walled carbon nanotubes. Phys. Rev. B 2009, 79, 233406. [CrossRef]

23. Duong, H.M.; Einarsson, E.; Okawa, J.; Xiang, R.; Maruyama, S. Thermal degradation of single-walled carbon nanotubes. Jpn. J. Appl. Phys. 2008, 47, 1994-1999. [CrossRef]

24. Zhang, W.; Deng, S.; Hong, T.; Zhang, Y. Design and Characteristics of a Vertical Carbon Nanotube Sheet Field Emitter. In Proceedings of the 33rd International Vacuum Nanoelectronics Conference (IVNC), Lyon, France, 6-7 July 2020 ; pp. 113-114. [CrossRef]

25. Lednev, V.N.; Pershin, S.M.; Obraztsova, E.D.; Kudryashov, S.I.; Bunkin, A.F. Single-shot and single-spot measurement of laser ablation threshold for carbon nanotubes. J. Phys. D Appl. Phys. 2013, 46, 052002. [CrossRef]

26. Rao, A.M.; Richter, E.; Bandow, S.; Chase, B.; Eklund, P.C.; Williams, K.A.; Fang, S.; Subbaswamy, K.R.; Menon, M.; Thess, A.; et al. Diameter-Selective Raman Scattering from Vibrational Modes in Carbon Nanotubes. Science 1997, 275, 187-191. [CrossRef]

27. Gorodetskiy, D.V.; Kurenya, A.G.; Gusel'nikov, A.V.; Baskakova, K.I.; Smirnov, D.A.; Arkhipov, V.E.; Bulusheva, L.G.; Okotrub, A.V. Laser beam patterning of carbon nanotube arrays for the work of electron field emitters in technical vacuum (vol B262, 114691, 2020). Mater. Sci. Eng. B Adv. 2021, 271, 115294. [CrossRef]

28. Labunov, V.; Prudnikava, A.; Bushuk, S.; Filatov, S.; Shulitski, B.; Tay, B.K.; Shaman, Y.; Basaev, A. Femtosecond laser modification of an array of vertically aligned carbon nanotubes intercalated with Fe phase nanoparticles. Nanoscale Res. Lett. $2013,8,375$. [CrossRef]

29. Qi, X.; Ruan, X.F.; Pan, C.X. Graphitization of solid carbon nanofibers at an unexpectedly low temperature. Mater. Lett. 2007, 61, 4272-4275. [CrossRef]

30. Lim, Z.H.; Lee, A.; Lim, K.Y.Y.; Zhu, Y.W.; Sow, C.H. Systematic investigation of sustained laser-induced incandescence in carbon nanotubes. J. Appl. Phys. 2010, 107, 064319. [CrossRef]

31. Srivastava, S.K.; Vankar, V.D.; Rao, D.V.S.; Kumar, V. Enhanced field emission characteristics of nitrogen-doped carbon nanotube films grown by microwave plasma enhanced chemical vapor deposition process. Thin Solid Films 2006, 515, 1851-1856. [CrossRef]

32. Jang, J.W.; Lee, C.E.; Lyu, S.C.; Lee, T.J.; Lee, C.J. Structural study of nitrogen-doping effects in bamboo-shaped multiwalled carbon nanotubes. Appl. Phys. Lett. 2004, 84, 2877-2879. [CrossRef]

33. Ago, H.; Kugler, T.; Cacialli, F.; Salaneck, W.R.; Shaffer, M.S.P.; Windle, A.H.; Friend, R.H. Work functions and surface functional groups of multiwall carbon nanotubes. J. Phys. Chem. B 1999, 103, 8116-8121. [CrossRef]

34. Hammer, P.; Victoria, N.M.; Alvarez, F. Effects of increasing nitrogen concentration on the structure of carbon nitride films deposited by ion beam assisted deposition. J. Vac. Sci. Technol. A 2000, 18, 2277-2287. [CrossRef]

35. Chan, L.H.; Hong, K.H.; Xiao, D.Q.; Hsieh, W.J.; Lai, S.H.; Shih, H.C.; Lin, T.C.; Shieu, F.S.; Chen, K.J.; Cheng, H.C. Role of extrinsic atoms on the morphology and field emission properties of carbon nanotubes. Appl. Phys. Lett. 2003, 82, $4334-4336$. [CrossRef]

36. Wei, G. Emission property of carbon nanotube with defects. Appl. Phys. Lett. 2006, 89, 143111. [CrossRef]

37. Kumar, A.; Parveen, S.; Husain, S.; Zulfequar, M.; Harsh; Husain, M. Oxygen and nitrogen doping in single wall carbon nanotubes: An efficient stable field emitter. J. Alloy Compd. 2017, 711, 85-93. [CrossRef]

38. Cai, D.; Liu, L. The screening effects of carbon nanotube arrays and its field emission optimum density. AIP Adv. 2013, 3, 122103. [CrossRef] 